Modern Physics Letters A

Vol. 18, No. 30 (2003) 2147-2148

(C) World Scientific Publishing Company

\title{
ERRATA
}

\section{SUPERFIELD APPROACH TO A NOVEL SYMMETRY FOR NON-ABELIAN GAUGE THEORY}

\author{
[Mod. Phys. Lett. A, Vol. 17, No. 3 (2002) 185-196]
}

R. P. Malik

The last sentence above Eq. (4.2) (p. 192) up to the line above Eq. (4.7) (p. 193) should read:

The Hodge duality $\star$ operation on the super differentials $\left(d Z^{M}\right)$ and their wedge products $\left(d Z^{M} \wedge d Z^{N}\right),\left(d Z^{M} \wedge d Z^{N} \wedge d Z^{P}\right)$ etc., defined on this supermanifold, is

$$
\begin{aligned}
& \star\left(d x^{\mu}\right)=\varepsilon^{\mu \nu}\left(d x_{\nu} \wedge d \theta \wedge d \bar{\theta}\right), \quad \star(d \theta)=\frac{1}{2 !} \varepsilon^{\mu \nu}\left(d x_{\mu} \wedge d x_{\nu} \wedge d \bar{\theta}\right), \\
& \star(d \bar{\theta})=\frac{1}{2 !} \varepsilon^{\mu \nu}\left(d x_{\mu} \wedge d x_{\nu} \wedge d \theta\right), \quad \star\left(d x^{\mu} \wedge d x^{\nu}\right)=\varepsilon^{\mu \nu}(d \theta \wedge d \bar{\theta}), \\
& \star\left(d x^{\mu} \wedge d \theta\right)=\varepsilon^{\mu \nu}\left(d x_{\nu} \wedge d \bar{\theta}\right), \quad \star\left(d x^{\mu} \wedge d \bar{\theta}\right)=\varepsilon^{\mu \nu}\left(d x_{\nu} \wedge d \theta\right), \\
& \star(d \theta \wedge d \theta)=\frac{1}{2 !} s^{\theta \theta} \varepsilon^{\mu \nu}\left(d x_{\mu} \wedge d x_{\nu}\right), \quad \star(d \theta \wedge d \bar{\theta})=\frac{1}{2 !} s^{\theta \bar{\theta}} \varepsilon^{\mu \nu}\left(d x_{\mu} \wedge d x_{\nu}\right), \\
& \star(d \bar{\theta} \wedge d \bar{\theta})=\frac{1}{2 !} s^{\bar{\theta} \bar{\theta}} \varepsilon^{\mu \nu}\left(d x_{\mu} \wedge d x_{\nu}\right), \quad \star\left(d x_{\mu} \wedge d \theta \wedge d \bar{\theta}\right)=\varepsilon_{\mu \nu}\left(d x^{\nu}\right), \\
& \star\left(d x_{\mu} \wedge d x_{\nu} \wedge d \theta \wedge d \bar{\theta}\right)=\varepsilon_{\mu \nu}, \quad \star\left(d x_{\mu} \wedge d x_{\nu} \wedge d \theta\right)=\varepsilon_{\mu \nu}(d \bar{\theta}), \\
& \star\left(d x_{\mu} \wedge d x_{\nu} \wedge d \bar{\theta}\right)=\varepsilon_{\mu \nu}(d \theta), \quad \star\left(d x_{\mu} \wedge d x_{\nu} \wedge d \theta \wedge d \theta\right)=\varepsilon_{\mu \nu} s^{\theta \theta},
\end{aligned}
$$

where $s$ 's are symmetric (e.g., $s^{\theta \bar{\theta}}=s^{\bar{\theta} \theta}$ etc.). In the above we have collected only a few of the $\star$ operations. The other $\star$ operations can be computed in an analogous 
manner. With these as the backdrop, we obtain the expression for the superscalar superfield $\tilde{\delta} \tilde{A}=-\star \tilde{d} \star \tilde{A}$ as

$$
\tilde{\delta} \tilde{A}=\left(\partial_{\mu} B^{\mu}\right)+s^{\theta \theta}\left(\partial_{\theta} \Phi\right)+s^{\bar{\theta} \bar{\theta}}\left(\partial_{\bar{\theta}} \bar{\Phi}\right)+s^{\theta \bar{\theta}}\left(\partial_{\theta} \bar{\Phi}+\partial_{\bar{\theta}} \Phi\right) .
$$

It will be noted that we have dropped all the terms in the computation of $(\tilde{d} \star \tilde{A})$ which contain (i) more than two differentials in spacetime, and (ii) more than two differentials in Grassmannian variables. Only after this, we have applied another $\star$ operation on it. Now we exploit the analogue of the horizontality condition w.r.t. $\tilde{\delta}$ (i.e. $\tilde{\delta} \tilde{A}=\delta A$ ) which is nothing but equating the R.H.S. of Eqs. (4.1) and (4.3). In other words, we set the codfficients of $s^{\theta \theta}, s^{\bar{\theta} \bar{\theta}}, s^{\theta \bar{\theta}}$ equal to zero. The ensuing restrictions on the superfields are

$$
\partial_{\theta} \bar{\Phi}+\partial_{\bar{\theta}} \Phi=0, \quad \partial_{\theta} \Phi=0, \quad \partial_{\bar{\theta}} \bar{\Phi}=0 .
$$

Exploiting the expansions (3.2), it can be checked that the above restrictions lead to

$$
s^{a}(x)=0, \quad \bar{s}^{a}(x)=0, \quad \mathcal{B}^{a}(x)+\overline{\mathcal{B}}^{a}(x)=0, \quad B^{a}(x)=\bar{B}^{a}(x)=0,
$$

and the following conditions on the component fields of $B_{\mu}(x, \theta, \bar{\theta})$

$$
\partial \cdot \bar{R}=0, \quad \partial \cdot R=0, \quad \partial \cdot S=0 .
$$

In terms of solutions (4.5) and $R_{\mu}^{a}=-\varepsilon_{\mu \nu} \partial^{\nu} \bar{C}^{a}, \bar{R}_{\mu}^{a}=-\varepsilon_{\mu \nu} \partial^{\nu} C^{a}, S_{\mu}^{a}=-\varepsilon_{\mu \nu} \partial^{\nu} \mathcal{B}^{a}$, the superfield expansion (3.2) can be re-expressed as 